\title{
Motivation para la actividad fisica e su relation con la calidad de vida
}

\author{
Motivation for physical adivity and its relation with the quality of life
}

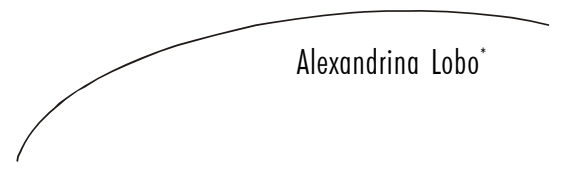

\section{Resumo}

Objetivos: Describir y analizar los motivos personales para practicar actividad física de los ancianos institucionalizados; determinar las asociaciones entre eses motivos y los niveles de calidad de vida y actividad física. Material y métodos: 185 ancianos participaran en un estudio de año con 4 programas de intervención: programa de ejercicio aerobio, treno de fuerza, educación de salud y grupo de control. Fueran evaluados los dados antropométricos (IMC), los niveles de actividad física (MTI actigraph), los motivos personales para practicar ejercicio (AMPEF) y la calidad de vida (SF-36) en 3 momentos (Línea de base, 1 año y 18 meses de seguimiento). Resultados: La motivación personal para el ejercicio después de la intervención es mayor en el grupo de ejercicio aeróbico $(2,9 \pm 0,5)$ y menor en el grupo de control $(2,3 \pm 0,6)$. En los 18 meses de seguimiento solo el grupo de control aumentó los niveles de motivación. Los niveles de motivación tienen mejor correlación con la componente física de la calidad de vida. Conclusión: Los motivos para la práctica de ejercicio físico en los ancianos institucionalizados son debidos fundamentalmente a razones de forma física y motivos interpersonales al cabo de 1 año y debido a razones psicológicas, de salud y de forma física al cabo de 18 meses de seguimiento.

Centro de Investigación de Actividad Física, Salud y Laser de la Facultad de Ciencias del Deporte y Educación Física de la Universidad de Oporto. Portugal

Correspondencia / Correspondence

Alexandrina Lobo

Av. Padre Júlio Fragata no 109, 3.1: S. Victor

4700413 - Braga, Portugal

E-mail: damiaolobo@gmail.com

\section{Palabras Clave:}

Salud del Anciano Institucionalizado. Ancianos. Motivación. Actividad Física. Ejercicio. Calidad de Vida. Educación en Salud. Índice de Masa Corporal. 


\section{Abstract}

Objective: To describe and analyze the motivations to the exercise; to determine the associations between theses motivations, levels of physical activity and quality of life. Measure: 185 elders participated in a one-year study with 4 intervention programs: aerobic exercise program, strength training, health education and control group. The anthropometric measures, levels of physical activity and quality of life were evaluated in 3 times (Baseline, 1 year and 18 months of follow-up). Results: The personal motivation for the exercise after the intervention is greater in the group of aerobic exercise $(2.9 \pm 0.5)$ and lower in the control group $(2.3 \pm 0.6)$; Within 18 months of follow-up only

Key words: Health of Institutionalized Elderly. Elderly. Motivation. Physical Activity. Exercise. Quality of Life. Health Education. Body Mass Index. the control group increased levels of motivation. The motivation levels are better correlated with the physical component of quality of life. Conclusion: The reasons for physical exercise in institutionalized elders are mainly due to reasons of fitness and interpersonal reasons in 1-year follow-up and by reasons of health, psychological and fitness in 18-month follow-up.

\section{INTRODUCCIÓN}

Los ancianos constituyen el sector de la población que más está creciendo en los últimos años. En Portugal, según los datos del Instituto Nacional de Estadística, los más de $8 \%$ ancianos en 1960 se han convertido en más de $16 \%$ en $2001 .{ }^{1}$ Hasta 2050, el Índice de Envejecimiento podrá situarse en los 398 ancianos por cada 100 jóvenes. ${ }^{2}$

Las personas mayores sufren un proceso en que se acentúa la progresiva involución en las cualidades físicas. El 85\% de los ancianos de Portugal llevan una vida sedentaria. La inmovilidad en el anciano es una de las entidades geriátricas más frecuentes y un factor de riesgo importante para los problemas cardiovasculares, y favorece patologías como el estreñimiento, la insuficiencia venosa, osteoporosis, etc. Está comprobado que la realización de ejercicio físico sua- viza y retrasa este proceso y, por tanto, mejora su calidad de vida. La realización de ejercicio físico por parte de estas personas tiene efectos beneficiosos para la salud como medida preventiva, de mantenimiento y rehabilitación. Asimismo, hay que considerar los aspectos recreativo y de relación social. ${ }^{3}$

El objeto de este estudio es examinar la relación entre los motivos personales para practicar ejercicio, los niveles de calidad de vida y actividad física.

La Teoría de la Autodeterminación ${ }^{4}$ constituye un modelo explicativo de la motivación humana y determina en qué medida las personas se involucran o no libremente en la realización de sus actividades, teniendo en cuenta una serie de mecanismos psicológicos reguladores de la conducta, y buscando en la medida de lo posible una mayor orientación hacia la motiva- 
ción autodeterminada. Los estudios muestran que dicha motivación autodeterminada está altamente relacionada con la motivación intrínseca, mientras que la motivación extrínseca y sus mecanismos reguladores favorecen conductas no autodeterminadas e incluso caracterizadas por la falta de motivación. Las implicaciones prácticas en el ámbito de la práctica de actividad física sugieren la conveniencia de desarrollar estrategias para desarrollar la motivación intrínseca y sus formas de regulación. ${ }^{5}$

\section{MATERIAL Y MÉTODOS}

\section{Diseño del estudio}

Este es un estudio longitudinal de 185 ancianos institucionalizados del área metropolitana de Oporto (Portugal). Los criterios de inclusión fueran: un buen estado cognitivo definido por una puntuación en el Mini-Mental State Examination (MMSE) > 24 puntos $^{6}$ y la capacidad de realizar los ejercicios propuestos. ${ }^{7}$ Se empleó una técnica de muestreo aleatorio simple para crear los programas de intervención: programa de ejercicio aerobio (EA), programa de treno de fuerza (TF), programa de educación de salud (ES) y grupo de control (GC).

\section{Sujetos y medidas}

En Enero de 2006 (línea de base) 185 ancianos (95 mujeres e 90 hombres) fueron seleccionados e invitados a participar en el estudio. Todos firmaran el consentimiento informado, de libre y esclarecida voluntad y demostraran comprensión por los objetivos del estudio y los datos fueron obtenidos por un investigador. Durante el estudio murieron 23 personas, 1 salió de la institución y 13 han abandonado el programa de intervención. La muestra final incluyó 148 sujetos. Los programas de intervención fueron de 1 año (segunda evaluación en Marzo de 2007) de duración con dos sesiones de ejercicio por semana, 30-40 minutos por sesión en los tres grupos. Para la intervención se crearon grupos de sesiones de 46 ancianos.

En el programa de ejercicio aerobio $(\mathrm{n}=38)$ se recomendó un calentamiento mas prolongado con una duración de 10-15 minutos realizando estiramientos, ejercicios calisténicos y actividad aeróbica bajo impacto. Seguido de un periodo de entrenamiento para mejorar la capacidad física que fue formado por varios tipos de ejercicios: caminar, bailar, etc. Por fin un enfriamiento o relajación de 10-15 minutos.

En el programa de treno de fuerza $(\mathrm{n}=10)$ lo objetivo visa acondicionar grupos musculares concretos, bien por desadaptación previa importante, o con la finalidad de mejorar la fuerza muscular necesaria para la ejecución de las actividades de vida diaria. Los ejercicios fueran con pesos de 1 a 2 kilos e incluso con aparatos de entrenamiento de resistencia.

En el programa de educación de salud $(n=43)$, los profesionales reforjaban y explicaban la necesidad de realizar ejercicio físico, 
indicando las posibilidades existentes en las instituciones y por ultimo los sujetos del grupo de control $(\mathrm{n}=57)$ fueron instruidos para mantener sus actividades de vida diaria y no fue levada a cabo ninguna intervención.

Después, en Septiembre2007, se realizó una tercera evaluación (18 meses de seguimiento). Se determinó el peso y la talla de acuerdo a los estándares habituales, con el objetivo de calcular el índice de masa corporal (IMC) mediante la fórmula peso/altura ${ }^{2}\left(\mathrm{en} \mathrm{kg} / \mathrm{m}^{2}\right)$; se determinó exceso de peso a las cifras mayores de $25 \mathrm{y}$ obesidad a las mayores de 30.

El SF-36 ${ }^{8}$ se utilizó para medir la percepción de la calidad de vida; esta es una medida genérica de salud, ya que se destina a medir conceptos de salud que representan valores humanos básicos relevantes, el estado funcional y el bienestar individual. Presenta 8 dimensiones: función física, limitaciones de rol por problemas físicos, dolor corporal, salud general, vitalidad, función social, limitaciones de rol por problemas emocionales y salud mental. En cada dominio las puntuaciones varían entre 0 (peor salud) y 100 puntos (mejor salud).

La evolución de los motivos personales para practicar ejercicio se determinó mediante el Autoinforme de Motivos para la Práctica de Ejercicio Físico (AMPEF). ${ }^{9} \mathrm{El}$ AMPEF evalúa 5 áreas generales relacionadas con la motivación para practicar ejercicio físico: A) Motivos Psicológicos; B) Motivos Interpersonales; C) Motivos de Salud; D) Motivos relacionados con el cuerpo; y E) Motivos de Forma Física. Cada una de estas áreas consta de diversos factores o escalas $(2,3$ o 4$)$ en un total de 14 factores. Este autoinforme no debe interpretarse en valor absoluto como el peso o importancia global de los motivos para practicar ejercicio físico, sino como un índice relativo de la evolución individual en los cambios de la motivación hacia el ejercicio. La puntuación puede oscilar entre 0 e 5 ( 0 se ese motivo no es nada cierto y 5 se considera ese motivo totalmente cierto).

El MTI Actigraph ${ }^{10}$ fue utilizado como una medida objetiva de la Actividad Física diario en siete días consecutivos. Para el presente estudio, la duración del Epoque se fijó en 1 minuto. Todos los participantes recibieron instrucciones sobre cómo utilizar los monitores. El tiempo dedicado diariamente a cada una de las categorías de la intensidad de actividad física se calculó de acuerdo con los puntos de corte de Freedson. ${ }^{11}$ Para la análisis, se utilizaran los minutos de actividad física moderada ya que la intensidad de la actividad física es importante para la promoción de la salud, de acuerdo con las recomendaciones del American College of Sports Medicine. ${ }^{12}$

\section{Análisis estadístico}

Tras efectuar un análisis descriptivo, se utilizó la prueba de la t de Student para comparar variables cuantitativas y la correlación de Pearson para verificar la existencia de asociaciones entre las diferentes variables. Para el tratamiento estadístico de los datos, se utilizó el programa SPSS 14.0, con 
un nivel de significación (intervalo de confianza [IC] del 95\%) $\mathrm{p}<0,05$.

\section{RESULTADOS}

En la evaluación inicial, los ancianos presentan tiempos de actividad física moderada similares y es el grupo de EA que tiene mejores valores de calidad de vida $(64,9 \pm 14,2)$, seguido del GC $(62,0 \pm 13,4)$ y en último el ES $(58,3 \pm 12,6)$. El IMC é mayor en el GC $(26,8 \pm 3,3)$, menor en el TF $(24,6 \pm 3,2)$ y non presenta alteraciones significativas al largo del tiempo.

Según los datos generales, los niveles de actividad física diminuyen entre las diferentes evaluaciones. En el grupo EA, entre la $2^{\mathrm{a}}$ e $3^{\mathrm{a}}$ evaluación los minutos de actividad física disminuyen significativamente, pero la cantidad total de actividad (counts) aumenta. Del mismo modo, la calidad de vida en la $3^{\mathrm{a}}$ evaluación también disminuye.

La calidad de vida del GC aumenta significativamente entre la segunda y tercera evaluación, lo que puede resultar de la oportunidad que tuvieran para realizar actividad física después del término de la intervención.

Hay que considerar que el grupo TF se destaca por mantener los niveles más altos de actividad física moderada y lo mismo en respecto a la cantidad total.

La motivación personal para el ejercicio después de la intervención es mayor en el grupo de EA $(2,9 \pm 0,5)$ y menor en el GC $(2,3 \pm 0,6)$. En la última evaluación, solo el GC aumentó los niveles, pero non hay diferencias estadísticamente significativas.

Tabla 1 - Características de los ancianos por grupos de intervención (media \pm desviación estándar). Oporto, Portugal, 2007.

\begin{tabular}{|c|c|c|c|c|c|c|}
\hline & & $\begin{array}{l}\text { Actividad Física } \\
\text { (minutos) }\end{array}$ & $\begin{array}{l}\text { Actividad Física } \\
\text { (counts) }\end{array}$ & $\begin{array}{c}\mathrm{IMC} \\
\left(\mathrm{kg} / \mathrm{m}^{2}\right)\end{array}$ & $\begin{array}{c}\text { SF-36 } \\
(\%)\end{array}$ & $\begin{array}{l}\text { AMPEF } \\
\text { (puntos) }\end{array}$ \\
\hline Grupo Control & Línea de base & $6,14 \pm 5^{b c}$ & $100474,5 \pm 76520,9^{\mathrm{ab}}$ & $26,8 \pm 3,3$ & $62,0 \pm 13,4$ & $\mathrm{X}$ \\
\hline \multirow[t]{2}{*}{$77,6 \pm 6,9$ años } & 1 año de seguimiento & $1,5 \pm 1,7^{\mathrm{ab}}$ & $42167,4 \pm 41589,3^{a}$ & $27,7 \pm 3,3$ & $58,2 \pm 10,4^{\mathrm{a}}$ & $2,3 \pm 0,6$ \\
\hline & $\begin{array}{l}18 \text { meses de } \\
\text { seguimiento }\end{array}$ & $0,6 \pm 3,5^{a c}$ & $82131,3 \pm 390304^{b}$ & $24,7 \pm 2,6$ & $62,3 \pm 13,7^{\mathrm{a}}$ & $2,4 \pm 0,5$ \\
\hline $\begin{array}{l}\text { Ejercicio } \\
\text { Aerobio }\end{array}$ & Línea de base & $6,9 \pm 5,2^{\mathrm{ab}}$ & $137414,7 \pm 89481,1^{a}$ & $25,6 \pm 3,2$ & $64,9 \pm 14,2^{b c}$ & $\mathrm{X}$ \\
\hline \multirow{2}{*}{$81,0 \pm 7,1$ años } & 1 año de seguimiento & $4,1 \pm 4,0^{a}$ & $92811,2 \pm 56707,5^{\mathrm{ab}}$ & $25,6 \pm 3,2$ & $70,1 \pm 6,9^{\mathrm{ab}}$ & $2,9 \pm 0,5$ \\
\hline & $\begin{array}{l}18 \text { meses de } \\
\text { seguimiento }\end{array}$ & $2,8 \pm 3,6^{b}$ & $120475,4 \pm 182033,5^{b}$ & $26,6 \pm 2,8$ & $62,5 \pm 13,6^{a c}$ & $2,4 \pm 0,7$ \\
\hline Treno de Fuerza & Línea de base & $7,34 \pm 6,2$ & $99598,9 \pm 60212,7$ & $24,6 \pm 3,2$ & $60,1 \pm 15,1^{\mathrm{ab}}$ & $\mathrm{X}$ \\
\hline \multirow[t]{2}{*}{$75,1 \pm 8,5$ años } & 1 año de seguimiento & $3,6 \pm 5,4$ & $65466,8 \pm 42526,7$ & $25,2 \pm 3,1$ & $68,4 \pm 11,1^{a}$ & $2,5 \pm 1,0$ \\
\hline & $\begin{array}{l}18 \text { meses de } \\
\text { seguimiento }\end{array}$ & $3,9 \pm 5,9$ & $89578,8 \pm 60056,8$ & $25,9 \pm 3,0$ & $67,9 \pm 10,5^{b}$ & $2,2 \pm 0,7$ \\
\hline Educación & Línea de base & & & & & $\mathrm{X}$ \\
\hline Salud & & $5,59 \pm 3,9^{\mathrm{bc}}$ & $132684,4 \pm 90792,4^{\mathrm{bc}}$ & $25,1 \pm 3,0$ & $58,3 \pm 12,6^{a b}$ & \\
\hline \multirow[t]{2}{*}{$81,6 \pm 6,7$ años } & 1 año de seguimiento & $0,6 \pm 1,9^{\mathrm{ab}}$ & $67876,5 \pm 39468,4^{\mathrm{ab}}$ & $25,6 \pm 2,7$ & $61,2 \pm 9,6^{a}$ & $2,5 \pm 0,6$ \\
\hline & $\begin{array}{l}18 \text { meses de } \\
\text { seguimiento }\end{array}$ & $1,7 \pm 2,9^{\mathrm{ac}}$ & $90595,3 \pm 50004,2^{\mathrm{ac}}$ & $26,0 \pm 2,9$ & $64,1 \pm 12,3^{\mathrm{b}}$ & $2,3 \pm 0,6$ \\
\hline
\end{tabular}

abc Prueba de la $\mathrm{t}$ de Student estatistica e significativamente diferentes entre los grupos.

$\mathrm{X}=$ non fue realizada la análisis 
En relación a los parámetros del autoinforme de los motivos para la práctica de ejercicio físico (tabla 2), verificamos que disminuyen entre las evaluaciones en todos los grupos. Analizando detalladamente, esa diferencia es significativa en el GC para los motivos psicológicos y de salud, en el grupo de EA, para los motivos psicológicos y de forma física, en el grupo de TF para los motivos interpersonales y en el grupo de ES para los motivos psicológicos. Lo que se refiere a los motivos relacionados con el cuerpo, verificamos que aumentan en todolos grupos entre las evaluaciones pero non de forma estadísticamente significativa. Y lo mismo se constata en los motivos de la forma física para el GCy ES.

Tabla 2 - Parámetros del Autoinforme de Motivos para la Práctica de Ejercicio Físico (media \pm desviación estándar). Oporto, Portugal, 2007.

\begin{tabular}{llcccccc}
\hline & & Psicológicos & Interpersonales & Salud & Forma Física & Corporais & AMPEF \\
\hline Grupo & & & & & & & \\
Control & 1 año de seguimiento & $1,91 \pm 0,83^{\mathrm{b}}$ & $1,95 \pm 0,78$ & $2,83 \pm 1,24^{\mathrm{a}}$ & $2,92 \pm 0,86$ & $2,07 \pm 0,59$ & $2,35 \pm 0,56^{\mathrm{a}}$ \\
& 18 meses de seguimiento & $1,63 \pm 0,95^{\mathrm{b}}$ & $1,92 \pm 0,83$ & $1,95 \pm 0,78^{\mathrm{a}}$ & $2,99 \pm 1,18$ & $2,82 \pm 0,82$ & $2,07 \pm 0,59^{\mathrm{a}}$ \\
& & & & & & & \\
Ejercicio & & & & & & \\
Aeróbico & 1 año de seguimiento & $2,21 \pm 0,76^{\mathrm{b}}$ & $2,79 \pm 1,23$ & $3,34 \pm 1,10$ & $3,82 \pm 0,96^{\mathrm{b}}$ & $2,43 \pm 0,76$ & $2,94 \pm 0,73^{\mathrm{b}}$ \\
& 18 meses de seguimiento & $1,62 \pm 0,92^{\mathrm{b}}$ & $2,21 \pm 0,76$ & $2,79 \pm 1,22$ & $3,46 \pm 1,07^{\mathrm{b}}$ & $3,82 \pm 0,99$ & $2,43 \pm 0,76^{\mathrm{b}}$ \\
& & & & & & & \\
Treno de & & & & & & & \\
Fuerza & 1 año de seguimiento & $1,57 \pm 0.99$ & $2,26 \pm 1,33^{\mathrm{a}}$ & $2,86 \pm 1,59$ & $3,03 \pm 1,14$ & $2,44 \pm 1,13$ & $2,53 \pm 1,01^{\mathrm{b}}$ \\
& 18 meses de seguimiento & $1,48 \pm 1,02$ & $1,57 \pm 0,99^{\mathrm{a}}$ & $2,56 \pm 1,38$ & $3,02 \pm 1,62$ & $3,03 \pm 1,26$ & $2,44 \pm 1,13^{\mathrm{b}}$ \\
& & & & & & & \\
Educación & & & & & & & \\
Salud & 1 año de seguimiento & $1,93 \pm 0,96^{\mathrm{a}}$ & $2,03 \pm 0,67$ & $3,03 \pm 1,04$ & $2,91 \pm 0,85$ & $2,15 \pm 0,5$ & $2,45 \pm 0,59^{\mathrm{a}}$ \\
& 18 meses de seguimiento & $1,28 \pm 0,95^{\mathrm{a}}$ & $1,93 \pm 0,96$ & $2,03 \pm 0,67$ & $3,17 \pm 1,03$ & $2,98 \pm 0,84$ & $2,15 \pm 0,5^{\mathrm{a}}$ \\
\hline
\end{tabular}

a - Prueba de la t de Student: $\mathrm{p}<0,05$. - b Prueba de la t de Student: $\mathrm{p}<0,01$.

En la tabla 3 se muestra el estudio de correlación entre las distintas variables estudiadas. Destacase una asociación fuerte y positiva $(\mathrm{p}$ $<0,05)$ entre la actividad física pasado un año de seguimiento con los motivos interpersonales $(r=0,724)$ y de la forma física $(r=0,654)$.
Después de 18 meses de seguimiento, la actividad física se correlaciona más fuertemente con los motivos de salud $(\mathrm{r}=0,817)$ y los motivos psicológicos $(r=0,712)$. 
Tabla 3 - Correlaciones entre la actividad física y las variables estudiadas. Oporto, Portugal, 2007.

\begin{tabular}{|c|c|c|c|c|c|c|c|c|c|}
\hline & Psicológicos & Interpersonales & Salud & $\begin{array}{c}\text { Forma } \\
\text { Física }\end{array}$ & Corporais & AMPEF & SF-36 & $\begin{array}{c}\text { SF- } \\
\text { Física }\end{array}$ & $\begin{array}{c}\text { SF- } \\
\text { Mental }\end{array}$ \\
\hline $\begin{array}{l}\text { Actividad Física } \\
1 \text { año de } \\
\text { seguimiento }\end{array}$ & $0,271^{* *}$ & $0,724^{* *}$ & $\begin{array}{c}0,323^{*} \\
*\end{array}$ & $0,654^{* *}$ & $0,454^{* *}$ & $0,593^{* *}$ & $0,264^{* *}$ & $0,188^{*}$ & $0,265^{* *}$ \\
\hline $\begin{array}{l}\text { Actividad Física } \\
18 \text { meses } \\
\text { seguimiento }\end{array}$ & $0,712^{* *}$ & 0,032 & $\begin{array}{c}0,817^{*} \\
*\end{array}$ & $0,658^{* *}$ & 0,044 & $0,751^{* *}$ & $0,380^{* *}$ & $\begin{array}{c}0,392^{*} \\
*\end{array}$ & $0,299^{* *}$ \\
\hline
\end{tabular}

**Correlación de Pearson: $\mathrm{p}<0,01$

*Correlación de Pearson: $\mathrm{p}<0,05$

Las correlaciones entre los puntos de los motivos para la práctica de ejercicio físico y la calidad de vida son pequeñas. Constatase que la actividad física tiene una correlación más fuerte con la calidad de vida en los 18 meses de seguimiento y destacase en su componente física.

Tabla 4 - Correlaciones entre la motivación y la calidad de vida. Oporto, Portugal, 2007.

\begin{tabular}{lccc}
\hline & SF-36 & SF-Física & SF-Mental \\
\hline AMPEF & $0,277^{* *}$ & $0,263^{*}$ & $0,210^{*}$ \\
1 año de seguimiento & $0,327^{* *}$ & $0,366^{* *}$ & $0,236^{* *}$ \\
$\begin{array}{l}\text { AMPEF } \\
18 \text { meses de }\end{array}$ & & \\
seguimiento & & \\
$* *$ Correlación de Pearson: $\mathrm{p}<0,01$ & & \\
*Correlación de Pearson: $\mathrm{p}<0,05$ & &
\end{tabular}

\section{DISCUSIÓN}

El nivel cultural y la forma de ser influyen poderosamente en la longevidad y pueden determinar comportamientos y actitudes saludables. La motivación humana tiene relación con el desarrollo y funcionamiento de la personalidad dentro de los contextos sociales, como describe la Teoría de la Autodeterminación. Esta teoría anali- za el grado en que las personas realizan sus acciones al nivel más alto de reflexión y se comprometen en las acciones con un sentido de elección. ${ }^{13}$

Confirmando lo descrito en la literatura científica, ${ }^{14,15}$ nuestros resultados indican que los niveles de actividad física después de un año de seguimiento están más correlacionados con razones de forma física y 
motivos interpersonales, aunque con 18 meses de seguimiento las ganancias de salud y una mejoría de los motivos psicológicos están más correlacionados con la actividad física. El aumento de la motivación intrínseca para el ejercicio fue el más fuerte preeditor de los resultados a más largo plazo entre las variables analizadas. La teoría de libre determinación describe la motivación intrínseca de una persona como la satisfacción general de participar en una actividad determinada en termos de la propia actividad.

Estos hallazgos apoyan la tesis de que algunos motivos para la participación en el ejercicio pueden ser percibidos como de control interno. Específicamente, un enfoque en el manejo de peso y la mejoría de la percepción de salud aumentan las presiones para la actividad física. Por su vez, los motivos internos para el ejercicio, en concreto motivos psicológicas y de disfrute social de afiliación son razones que aumentan la percepción de la libre determinación y conducen a un mayor disfrute ejercicio. ${ }^{15}$

El sedentarismo en las mujeres adultas se debe, en la mayoría de los casos, a que suelen disponer de menos tiempo libre. “Asumen las tareas domésticas, el cuidado de la familia y, en general, tienden a percibir la actividad física como algo menos importante. Contodo la porcentaje de actividad se iguala entre sexos a partir de los 65 años, etapa en la que los hijos ya han abandonado el núcleo familiar o están jubiladas, disponiendo de más tiempo libre."16

Maltby, ${ }^{17}$ en un estudio con jóvenes, concluyo que para él que hace ejercicio hace menos de 6 meses, las motivaciones extrín- secas de ejercicio fueron significativamente más pobres en relación con el bienestar psicológico. Y para las personas que ejercen por 6 meses o más, las motivaciones intrínsecas se relacionaron significativamente con un mejor bienestar psicológico.

De acuerdo con la teoría de la evaluación cognitiva, ${ }^{18}$ la satisfacción de sentirse autónomo y competente estimula la motivación intrínseca, y los incentivos externos pueden afectar a las experiencias de autodeterminación, cambiando la percepción del locus de causalidad de interno "lo hago porque quiero" a externo "lo hago porque otros quieren”. Cuando los sujetos perciben que su conducta está controlada por los incentivos externos, disminuye el sentido de autonomía y, por lo tanto, la motivación intrínseca. Sin embargo, también se señala que si el incentivo se percibe como información de competencia o de progreso en la tarea, más que controlador de la conducta, puede aumentar la motivación intrínseca. ${ }^{19,20}$ Y cuando los individuos están más motivados intrínsecamente, experimentando elección, hay la ausencia de presión y mayor disfrute de la actividad.

Los tipos de motivación extrínsecos son los contingentes que llegan a una meta, pero están reguladas internamente, y son considerados intrínsecamente agradables e interesantes por la persona. ${ }^{21}$ Es importante destacar que de acuerdo con esta teoría, participación en actividades en que están intrínsecamente motivados (o bien integrados con los valores) y non siendo controlados externamente es importante para satis- 
facer las necesidades humanas y fundamental para la competencia y autonomía. ${ }^{17}$

La calidad de vida incluye de una manera complexa la salud física de una persona, su estado psicológico, sus sentimientos, sus relaciones sociales y comportamientos relacionados con su funcionamiento diario. Según Rikli y Jones, ${ }^{22}$ el envejecimiento tiene efectos negativos, limitando la su capacidad de realizar las actividades de la vida diaria y mantener la calidad de vida. Algunos estudios revelan que un de los más importantes aspectos relacionados con la salud y funcionalidad de la populación anciana puede ser representada por su autonomía. ${ }^{23}$

Estés resultados sugieren quela participación en la actividad física está directamente relacionada con la motivación, en especial en el grupo de ejercicio aeróbico. Los resultados también indican que la práctica de ejercicio tiene implicaciones en la calidad de vida, especialmente en relación a su componente física. La actividad física es un elemento que condiciona la calidad de vida y por tanto, la salud y bienestar.

\section{CONCLUSIÓN}

Estes resultados sugieren que los motivos para la práctica de ejercicio físico son debidos fundamentalmente a razones de forma física y motivos interpersonales al cabo de la intervención. Y después debido a motivos psicológicos, de salud y de forma física.

Tiendo en cuenta estes resultados, sugierese la implementación de programas que estimulen este colectivo a tener una vida más activa, llevando a cabo políticas sociales dirigidas a mejorar sus instalaciones, la accesibilidad, con personal especializado y mayor implicación de los profesionales.

\section{AGRADECIMIENTOS}

A las instituciones que autorizaron la recolecta de datos y en particular a las personas evaluadas en este estudio que han manifestado mucha fuerza de voluntad en superarse a sí mismas. A todos muchas gracias. 


\section{REFERÊNCIAS}

1. Instituto Nacional de Estatística. Censos. Resultados do XIV recenseamento geral da população: IV recenseamento geral da Habitação. Lisboa: 2001. Disponível em: URL : < http://censos.ine.pt/xportal/ xmain? $x$ pid $=$ CENSOS\& $x$ pgid $=$ ine_censos_publicacao_det\&menuBO $\mathrm{UI}=13707294 \&$ contexto $=$ pu\&PUBLICAC OESpub_boui $=379028 \&$ PUBLICACOESmodo $=2 \& \operatorname{sel} T a b=t a b 1>$.

2. Instituto Nacional de Estatística. Projecções de população residente, Portugal e NUTSII: decréscimo e envelhecimento da população até 2050. Lisboa: 2004. Disponível em : URL : < http://www.ine.pt/xportal/ xmain?xpid=INE\&xpgid=ine_desta ques\&DESTAQUESdest_boui $=72193 \&$ DESTAQUESmodo $=2>$.

3. Peral R, Ruipérez I, ed. La jubilación saludable. Ejercicio físico. In : López A. (ed.) La Jubilación. 1999, Fundación Promedic: Barcelona. 289-333.

4. Deci EL, Ryan RM. Intrinsic Motivation and Self-Determination in Human Behavior. New York : Plenum. 1985. 371 p.

5. Moreno JA, Martínez A. Importancia de la Teoría de la Autodeterminación en la práctica físico-deportiva: fundamentos e implicaciones prácticas. Cuadernos de Psicología del Deporte. 2006; 6(2): 39-54.

6. Folstein MF, Folstein SE, McHugh PR. Mini-Mental state a practical method for grading the cognitive state of patients for the clinician. J Psychiatr Res 1975; 12: 189-98.

7. Wade D, Collin C. The Barthel ADL Index : a standard measure of physical disability? Int Disabil Stud 1988; 10: 64-7.

8. Ferreira P. Criação da versão Portuguesa do MOS SF-36: Parte I- Adaptação cultural e linguística. Acta Med Port 2000; 13: 55-63.

9. Capdevila Ll, Niñerola J, Pintanel M. Motivación y actividad física: el autoinforme de Motivos para la práctica del ejercicio físico (AMPEF). Revista de Psicología del Deporte. 2004; 13(1): 55-74.

10. Reilly J, et al. Validation of actigraph accelerometer estimates of total energy expenditure in young children. Int J Pediatr Obes 2006; 1(3): 161 - 7.

11. Freedson PS, Melanson E, Sirard J. Calibration of the computer science and applications, Inc. accelerometer. Med Sci Sports Exerc 1998; 30(5): 777-81.

12. Nelson, ME, et al. Physical activity and public health in older adults: recommendation from the American College of Sports Medicine and the American Heart Association. Med Sci Sports Exerc 2007, 39(8): 1435-45.

13. Deci EL, Ryan RM. The general causality orientations scale: self-determination in personality. J Res Pers 1985; 19: 109-34.

14. Sherwood NE, Jeffery RW. The behavioral determinants of exercise: implications for physical activity interventions. Annu Rev Nutr 2000; 20: 21-44.

15. Ryan RM, Deci EL. Self-determination theory and the facilitation of intrinsic motivation, social development, and wellbeing. Am Psychol 2000; 55(1): 68-78.

16. Sociedad Española de Medicina Familiar y Comunitaria (SemFYC). Estudio sobre promoción del ejercicio físico. Madrid, 2007 enero 11. [Acesso $2008 \mathrm{abr}$.] Disponível em: URL: < http:// www.mujerydeporte.org/documentos/ docs/Estudio\%20sobre_2007.pdf $>$. 
17. Maltby J, Day L. The relationship between exercise motives and psychological well-being. J Psychol 2001; 135: 651-60.

18. Markland D. Internamente informativos internos frente a ejercer el control de motivos y en el ejercicio de disfrute: el papel mediador de la libre determinación. En P. Parisi, F. Pigozzi, y Prinzi G. (Eds.). Sport Science'99 en Europa Actas del $4^{\circ}$ Congreso Anual del Colegio Europeo de Ciencias del Deporte Roma: Instituto Universitario de Ciencias de motor, 1999.

19. Chatzisarantis NLD, et al. The cognitive processes by which perceived locus of causality predicts participation in physical activity. J Health Psychol 2002; 7(6): 685-99.
20. Markland D, Hardy L. The exercise motivations inventory: preliminary development and validity of a measure of individuals' reasons for participation in regular physical exercise. Pers Individ Dif 1993; 15(3): 289-96.

21. Boyd MP, Weinmann C, Yin Z. The relationship of physical selfperceptions and goal orientations to intrinsic motivation for exercise. Journal of Sport Behavior. 2002; 25: 1-18.

22. Rikli R, Jones C. Development and validation of a functional fitness test for community-residing older adults. Journal of aging and Physical Activity. 1999; 7: 129-61.

23. Jackson AS. The Evolution and Validity of Health-Related Fitness. Quest. 2006; 58: 160-75. 